\title{
Reference Equations for Impulse Oscillometry System Parameters in Healthy Brazilian Children and Adolescents
}

\author{
Maíra S de Assumpção MSc, Renata M Gonçalves MSc, Renata Martins MSc, \\ Tatiana G Bobbio PhD, and Camila IS Schivinski PhD
}

\begin{abstract}
BACKGROUND: The impulse oscillometry system is a noninvasive method of evaluating respiratory mechanics that is being increasingly used in the pediatric population. To date, no reference equations have been developed for healthy Brazilian children and adolescents. The purpose of the study was to determine the reference equations for impulse oscillometry system parameters in healthy children and adolescents. METHODS: We undertook an observational cross-sectional analytical study with healthy students age 6-14 y from educational institutions in the Greater Florianópolis area. Biometric data were evaluated (weight, height, body mass index, and body surface area) as predictors and for sample characterization. All participants were submitted to evaluation of respiratory mechanics using an oscillometry system following American Thoracic Society standards. Three tests were recorded, with data acquisition for $\geq 20 \mathrm{~s}$. For data normality verification, the Kolmogorov-Smirnov test was applied, and Pearson's correlation test identified the relationship between the predicted values of height, age, and weight and the oscillometric variables of resistance at 5 and $20 \mathrm{~Hz}$ reactance; respiratory impedance at $5 \mathrm{~Hz}$ resonant frequency; and reactance area. Models were developed using simple linear regression and multiple analyses. RESULTS: After the recruitment of 864 children, 123 subjects with an average age of $10.0 \pm 2.4 \mathrm{y}$ for boys and $9.9 \pm 2.4 \mathrm{y}$ for girls $(P=.94)$ were considered for final analysis. Correlations were identified between the dependent and predictor oscillometric variables, with height identified as having the greatest predictive power in the equations developed for boys in all oscillometric parameters, with a mean adjusted $R^{2}$ of $46.51 \%$. Age had greater influence on resonant frequency (adjusted $R^{2}=40.1 \%$ ) and reactance area (adjusted $R^{2}=48.8 \%$ ) for girls. CONCLUSIONS: Reference equations were developed for males and females, and height was the most influential predictor variable for most impulse oscillometry system parameters in the population studied. Key words: respiratory mechanics; oscillometry; reference values; children; pulmonary function test; regression analyses. [Respir Care 2016;61(8):1090-1099. () 2016 Daedalus Enterprises]
\end{abstract}

\section{Introduction}

The development of methods for the assessment of the respiratory system emerged with the aim of understanding

\footnotetext{
Ms de Assumpção, Ms Gonçalves, Ms Martins, and Dr Schivinski are affiliated with the Health and Sports Science Center (CEFID), Physiotherapy Department, Santa Catarina State University, Florianópolis, SC, Brazil. Dr Bobbio is affiliated with Texas A\&M University, College Station, Texas.

This work was supported by the Brazilian Federal Agency for Support and Evaluation of Graduate Education (CAPES) and the Brazilian National Council for Scientific and Technological Development (CNPq). The authors have disclosed no conflicts of interest.
}

lung physiology, growth, and development as well as verifying the association of these factors with changes in respiratory mechanics. ${ }^{1}$

The impulse oscillometry system is an important resource for the evaluation of such changes. ${ }^{2-4}$ The impulse oscillometry system has been successful, particularly in

\footnotetext{
Correspondence: Maíra Seabra de Assumpção, Rua Rio de Janeiro 1326, Enseada, São Francisco do Sul, SC 89240-000, Brazil. E-mail: mairaassumpcao@yahoo.com.br.
}

DOI: $10.4187 /$ respcare. 04226 
the respiratory assessment of children, because it does not require effort and it is easy to apply.,5-8

The literature presents some studies using the impulse oscillometry system as a complementary method of evaluation in children with respiratory disorders, with emphasis on asthma and cystic fibrosis. ${ }^{8-12}$ Investigations carried out with healthy children show a growing trend toward determining equations/reference values for new methods of respiratory assessment. ${ }^{13}$ The main studies on this subject were conducted in different countries, and, as yet, no equations/reference values for impulse oscillometry system parameters have been presented for healthy children in Brazil.

Recently, a publication addressed the determination of reference values for Mexican children and adolescents, ${ }^{14}$ also showing the use of this respiratory test in Latin American countries. This study, as well as other research in the area of pediatrics, shows that height is considered the most influential variable for impulse oscillometry system parameters and an important element for determining equations and reference values.

In Brazil, determining these equations will help to monitor lung function and respiratory symptoms in children and will enable comparisons between different pediatric populations. In addition, it is a useful tool to complement tests such as spirometry, which requires difficult forced maneuvers that could prevent the achievement of qualitatively and quantitatively satisfactory results. ${ }^{15}$ The present study aims to determine reference equations for impulse oscillometry system parameters in Brazilian children and adolescents age $6-14 \mathrm{y}$.

\section{Methods}

This is an observational cross-sectional analytical study with school-age children and adolescents, approved by the Research Ethics Committee of Universidade do Estado de Santa Catarina (UDESC), Brazil (approval number 97/2011). Data collection was conducted at public and private elementary schools of the Greater Florianópolis area from October 2012 to May 2014. After receiving authorization, the schools informed the child's guardian about the goals, procedures, risks, and benefits of the research, and informed consent was obtained.

Healthy children, age 6-14 y, participated in the study. Based on the guidelines of the American Thoracic Society and European Respiratory Society, individuals with the following characteristics were excluded: family history of wheezing or asthma, personal history of allergic rhinitis (ISAAC), ${ }^{16}$ premature birth, low birthweight or history of pulmonary invasive mechanical ventilation and/or bronchopulmonary dysplasia in the neonatal period, exposure to passive smoking in the family or during pregnancy, obesity according to the classification of the Telessaúde

\section{QUICK LOOK}

\section{Current knowledge}

The determination of reference equations for the impulse oscillometry system allows comparisons between different nationalities and extends the use of this tool for noninvasive evaluation of respiratory mechanics. As in other studies, height is identified as the most influential predictor variable for most impulse oscillometry parameters in children and adolescents.

\section{What this paper contributes to our knowledge}

Considering the impulse oscillometry parameters studied, 6 reference equations in Brazilian healthy children and adolescents were obtained for each sex. Height was identified as having the greatest predictive power in most of the equations developed for boys and girls.

Program of the Ministry of Health, recent upper respiratory tract infection (up to 2 weeks before evaluation), severe respiratory disease, and dyspnea. ${ }^{3,16-20}$ In addition, individuals were also excluded if they presented cardiorespiratory, musculoskeletal, neurological, or rheumatic diseases and auditory or visual deficits that might interfere with data collection and acquisition.

For the implementation of the data collection, all participants underwent biometric tests. Weight $(\mathrm{kg})$ was measured with a previously calibrated glass digital scale (Ultra Slim W903-Wiso, China). Height (inches/cm) was assessed by means of a portable Stadiometer (Personal Caprice Sanny - Fortaleza, CE/Brazil). Body mass index was calculated for each child as $\mathrm{kg} / \mathrm{m}^{2}$. Body surface area was also calculated based on the study of Du Bois et al..$^{21}$ Body surface area is routinely used in studies on impulse oscillometry in children. ${ }^{20,22}$ For the lung function tests (spirometry and impulse oscillometry), the MasterScreen impulse oscillometry system (Erich Jaeger, Friedberg, Germany) was used. Before each period of data collection, the system was calibrated using a digital thermo hygrometer and humidity monitor (model 7663, Incoterm/China).

Participants were trained on an individual basis at the time of collection, and each assessment lasted about 15-20 min. All tests were carried out by the same researcher. To ensure that there was no interference of the forced expiratory maneuvers with the results of the examination, an oscillometry test was carried out before the spirometry.

The oscillometry test was then carried out in accordance with American Thoracic Society guidelines following the same rules for the forced oscillation technique. ${ }^{3}$ The children were instructed to position the mouth on the mouthpiece and breathe spontaneously in tidal volume. A min- 
imum time of $20 \mathrm{~s}$ was set for data acquisition. Tests with changes in the oscillometric parameters during this collection were disregarded. Tests were only considered valid if the trace was linear, ascendant, and within the system's range of normality without interference, such as cough, crying, or swallowing, during measurements. ${ }^{20,23,24}$ Three measures were carried out, and each participant's best test was selected. A maximum of $10 \%$ variation between measurements was allowed for each of the parameters. To ensure test precision, coherence was measured at $5 \mathrm{~Hz}=0.8 \mathrm{~cm} \mathrm{H}_{2} \mathrm{O}$ and at $20 \mathrm{~Hz}=0.9 \mathrm{~cm} \mathrm{H}_{2} \mathrm{O}$.

For data analysis, the following parameters were considered: total airway resistance (resistance to $5 \mathrm{~Hz}$ [R5]), central airway resistance (resistance to $20 \mathrm{~Hz}$ [R20]), respiratory impedance (impedance to $5 \mathrm{~Hz}$ [Z5]), peripheral capacitive reactance (reactance to $5 \mathrm{~Hz}$ [X5]), reactance curve area below zero (AX), and resonant frequency (Fres). Frequencies of 5 and $20 \mathrm{~Hz}$ were used because they are the ones used most often for interpreting the presence of obstructive disorders. The oscillometric parameters measured at high frequency $(20 \mathrm{~Hz})$ express the behavior of large airways, whereas those measured at low frequency $(5 \mathrm{~Hz})$ reflect the behavior of the small airways. ${ }^{25}$ At the end of the oscillometric test, spirometry was carried out to verify the absence of disease. Participants were excluded if they presented changes in $\mathrm{FEV}_{1}$ and $\mathrm{FVC}$, with values below $80 \%$ of $\mathrm{FEV}_{1} / \mathrm{FVC}$ or below 0.70 of predicted. ${ }^{26}$ Those who did not show curves within the criteria of acceptability and reproducibility were also excluded. ${ }^{27}$ American Thoracic Society/European Respiratory Society guidelines were followed for the spirometric testing.

The sample calculation was carried out by determining the mean and $95 \% \mathrm{CI}$ of the impulse oscillometry system parameters according to sex. Thus, three impulse oscillometry system parameters were selected: R5, which expresses the total airway resistance; X5, representing the peripheral area of the airway; and Fres, which is a graphic variable identifying the presence of obstructive disorders.

The data for these parameters were obtained through a pilot study with 54 children and adolescents age 6-14 y. Based on the SD value of each parameter, a specific $\Delta$ was calculated according to the respective behavior of variation, and using the formula recommended by Hair et al, ${ }^{28}$ a minimum sample size of 96 individuals was considered sufficient. This result considered the highest value among the 3 chosen variables $(\mathrm{R} 5=\mathrm{SD}$ of 1.77 and $\Delta=0.5$ ). The formula given by Freund and Simon ${ }^{29}$ was also considered, according to which a minimum of 20 individuals is required for each predictor variable in studies on equations/reference values. Including the variables measured for the present study (height and age), a total of 80 participants ( 40 boys and 40 girls) was considered sufficient.

For statistical analysis, we evaluated the fit of the dependent variables to the normal distribution using the

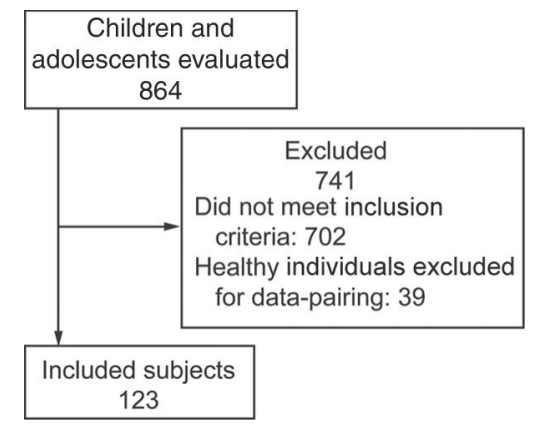

Fig. 1. Flow chart.

Kolmogorov-Smirnov test. In females, a base 10 transformation of the variables Z5, R5, R20, Fres, and AX was performed. In males, the variable $\mathrm{AX}$ was also transformed.

Pearson's correlation was used; therefore, simple linear regression analysis was carried out to evaluate the relationship between the impulse oscillometry system parameters (dependent variables) and the anthropometric data (predictor variables). ${ }^{30}$ Next, multivariate models were developed by multiple linear regression, incorporating predictor variables correlated to the dependent variable. The stepwise method was used to select the variables. The probability for inclusion of a predictor variable in the model was $P \leq .05$, and probability for exclusion was $P \geq .10$. The multicollinearity was evaluated among the predictor variables age, weight, height by variance inflation factor, and tolerance. For evaluation of model adjustment, residual analysis was carried out. The mean of the residuals was zero, and they had regular distribution. To choose the best model, the coefficient of determination (adjusted $\mathrm{R}^{2}$ ) and the theoretical reference on the topic were taken into account.

Data were analyzed using SPSS 20.0 for Windows (IBM Corp, Armonk, New York). The significance level was set at 5\% $(P \leq .05)$ for the Mann-Whitney test and $1 \%(P \leq .01)$ for the remaining tests.

\section{Results}

Initially, 864 children were evaluated from which 741 were excluded, for a total of 123 schoolchildren. Exclusion criteria included: allergic rhinitis, asthma, bronchitis, lung disease, passive smoking, history of pneumonia, respiratory infection, equipment failure, incomplete questionnaire, obesity, underweight, modified spirometries, prematurity, refused participation, other nationality, above age limit, and other adverse conditions (myelomeningocele, dwarfism, rheumatism, hearing impairment, attention deficit, diabetes, hypothyroidism, thoracic deformity). The same child or adolescent may have been excluded for multiple reasons (Figure 1). 
REFERENCE EQuATIONS FOR IMPULSE OsCILLOMETRY

Table 1. Mean \pm SD for Height, Weight, and Body Mass Index of the Children and Adolescents, Stratified by Sex and Age

\begin{tabular}{|c|c|c|c|c|c|c|c|c|}
\hline \multirow{2}{*}{ Age, y } & \multicolumn{4}{|c|}{ Boys } & \multicolumn{4}{|c|}{ Girls } \\
\hline & $n$ & Height, $\mathrm{cm}$ & Weight, kg & BMI (TS), kg/m² & $n$ & Height, cm & Weight, kg & BMI (TS), $\mathrm{kg} / \mathrm{m}^{2}$ \\
\hline 6 & 8 & $121.2 \pm 7.8$ & $23.4 \pm 3.9$ & $15.7 \pm 1.0$ & 7 & $116.8 \pm 4.4$ & $21.1 \pm 3.2$ & $15.2 \pm 1.4$ \\
\hline 7 & 5 & $123.9 \pm 3.6$ & $24.9 \pm 2.7$ & $16.2 \pm 1.7$ & 5 & $127.2 \pm 2.8$ & $26.4 \pm 1.7$ & $16.3 \pm 1.5$ \\
\hline 8 & 5 & $129.5 \pm 3.0$ & $26.7 \pm 3.2$ & $15.9 \pm 1.3$ & 5 & $130.3 \pm 9.4$ & $27.1 \pm 4.5$ & $15.9 \pm 2.2$ \\
\hline 9 & 6 & $132.9 \pm 9.6$ & $30.1 \pm 7.0$ & $16.8 \pm 1.9$ & 8 & $136.4 \pm 8.2$ & $34.0 \pm 6.3$ & $18.1 \pm 2.0$ \\
\hline 10 & 10 & $141.7 \pm 5.3$ & $35.0 \pm 6.6$ & $17.3 \pm 2.3$ & 10 & $147.6 \pm 8.2$ & $38.9 \pm 7.5$ & $17.7 \pm 2.2$ \\
\hline 11 & 7 & $148.5 \pm 5.7$ & $40.9 \pm 8.4$ & $18.4 \pm 2.8$ & 7 & $143.6 \pm 5.0$ & $45.2 \pm 7.1$ & $18.9 \pm 2.1$ \\
\hline 12 & 9 & $152.6 \pm 7.9$ & $45.9 \pm 9.8$ & $19.6 \pm 2.8$ & 9 & $149.6 \pm 7.6$ & $42.9 \pm 4.2$ & $19.1 \pm 2.4$ \\
\hline 13 & 7 & $162.3 \pm 8.1$ & $49.7 \pm 7.8$ & $19.0 \pm 2.0$ & 7 & $161.3 \pm 6.0$ & $49.4 \pm 5.3$ & $18.9 \pm 1.9$ \\
\hline 14 & 4 & $167.3 \pm 14.1$ & $57.3 \pm 13.9$ & $19.9 \pm 2.4$ & 4 & $161.1 \pm 2.8$ & $48.8 \pm 6.6$ & $18.9 \pm 2.3$ \\
\hline Total (123) & 61 & $142.2 \pm 16.1^{*}$ & $36.9 \pm 12.5^{*}$ & $17.7 \pm 2.5^{*}$ & 62 & $142.8 \pm 15.9 *$ & $37.2 \pm 10.9 *$ & $17.7 \pm 2.3^{*}$ \\
\hline
\end{tabular}

Table 2. Mean $\pm \mathrm{SD}$ for $\mathrm{FEV}_{1}, \mathrm{FVC}$, and $\mathrm{FEV}_{1} / \mathrm{FVC}$ of Children and Adolescents, Stratified by Sex and Age

\begin{tabular}{|c|c|c|c|c|c|c|}
\hline \multirow{2}{*}{ Age, y } & \multicolumn{3}{|c|}{ Boys } & \multicolumn{3}{|c|}{ Girls } \\
\hline & $\mathrm{FEV}_{1}, \% *$ & $\mathrm{FVC}, \% *$ & $\mathrm{FEV}_{1} / \mathrm{FVC}$ & $\mathrm{FEV}_{1}, \% *$ & $\mathrm{FVC}, \% *$ & $\mathrm{FEV}_{1} / \mathrm{FVC}$ \\
\hline 6 & $99.7 \pm 15.9$ & $94.8 \pm 12.0$ & $0.9 \pm 0.39$ & $99.6 \pm 9.7$ & $101.3 \pm 14.3$ & $0.9 \pm 0.06$ \\
\hline 7 & $99.3 \pm 10.2$ & $101.8 \pm 11.5$ & $0.8 \pm 0.10$ & $94.5 \pm 10.7$ & $98.4 \pm 14.5$ & $0.8 \pm 0.03$ \\
\hline 8 & $97.6 \pm 7.8$ & $97.3 \pm 14.7$ & $0.9 \pm 0.07$ & $95.5 \pm 7.1$ & $100.8 \pm 9.9$ & $0.8 \pm 0.03$ \\
\hline 9 & $102.2 \pm 10.0$ & $104.8 \pm 10.5$ & $0.8 \pm 0.02$ & $93.8 \pm 11.2$ & $100.1 \pm 7.8$ & $0.8 \pm 0.04$ \\
\hline 10 & $98.0 \pm 9.3$ & $101.4 \pm 7.0$ & $0.8 \pm 0.03$ & $93.3 \pm 9.7$ & $97.7 \pm 8.7$ & $0.9 \pm 0.04$ \\
\hline 11 & $96.4 \pm 4.9$ & $101.8 \pm 4.4$ & $0.8 \pm 0.05$ & $93.7 \pm 13.7$ & $98.4 \pm 12.2$ & $0.9 \pm 0.05$ \\
\hline 12 & $96.4 \pm 10.1$ & $97.9 \pm 9.7$ & $0.8 \pm 0.06$ & $96.6 \pm 8.6$ & $104.2 \pm 6.8$ & $0.8 \pm 0.05$ \\
\hline 13 & $98.7 \pm 8.2$ & $104.2 \pm 6.8$ & $0.8 \pm 0.05$ & $92.4 \pm 8.2$ & $98.5 \pm 8.7$ & $0.8 \pm 0.06$ \\
\hline 14 & $99.5 \pm 18.4$ & $108.4 \pm 11.8$ & $0.8 \pm 0.06$ & $89.1 \pm 5.5$ & $96.7 \pm 12.7$ & $0.8 \pm 0.10$ \\
\hline Total & $98.6 \pm 10.1$ & $101.1 \pm 9.8$ & $0.8 \pm 0.05$ & $94.6 \pm 9.5$ & $99.8 \pm 10.4$ & $0.8 \pm 0.05$ \\
\hline
\end{tabular}

We also excluded 39 children and adolescents who could not be paired for the statistical analysis in order to decrease selectivity bias and to make the sample as homogeneous as possible. $78 \%$ of the selected children were classified as normal-weight, and $94.3 \%$ were identified as white. Boys were age $10.0 \pm 2.4 \mathrm{y}$ on average, and girls were age $9.9 \pm 2.4$ y $(P=.94)$. Biometric characteristics of height, weight, and body mass index are presented in Table 1.The mean body surface areas were $1.20 \pm 0.26$ and $1.21 \pm 0.24 \mathrm{~m}^{2}(P=.83)$ for boys and girls, respectively.

According to the inclusion criteria, all participants had ISAAC ${ }^{16}$ scores below the cutoff point, with mean scores of $0.61 \pm 1.06$ and $0.74 \pm 1.25$ in the asthma component and $0.39 \pm 0.75$ and $0.46 \pm 0.72$ in the rhinitis component for boys and girls, respectively. All schoolchildren showed spirometry values within regular range. Mean values are shown in Table 2. Correlations were found between dependent oscillometry variables and predictor variables, as described in Table 3.

The results of the Pearson correlation between oscillometric parameters (R5, R20, X5, Z5, Fres, and AX) and the anthropometric data show that the variable height had the highest coefficients, ranging from 0.39 to 0.79 in boys and from 0.74 to 0.77 in girls. There were no differences between the sexes in the oscillometric variables studied (Table 4). Association of selected impulse oscillometry parameters with height and age is presented in Figure 2.

Table 5 presents the models of equations obtained by multiple linear regression analysis, according to the sex of the participants (boys and girls). Height was the variable with the most predictive power for models of all oscillometric variables in boys and for most of the variables in girls, with the highest coefficients of determination $\left(\mathrm{R}^{2}\right)$. 
Table 3. Mean for Resistance at $5 \mathrm{~Hz}$, Resistance at $20 \mathrm{~Hz}$, Reactance at $5 \mathrm{~Hz}$, Impedance at $5 \mathrm{~Hz}$, Resonant Frequency, and Reactance Curve Area Below Zero Stratified by Sex and Age

\begin{tabular}{|c|c|c|c|}
\hline IOS Parameters & Boy & Girl & $P$ \\
\hline \multicolumn{4}{|l|}{ Age $6 y$} \\
\hline $\mathrm{R} 5, \mathrm{kPa} / \mathrm{L} / \mathrm{s}$ & 0.730 & 0.746 & $>.99$ \\
\hline $\mathrm{R} 20, \mathrm{kPa} / \mathrm{L} / \mathrm{s}$ & 0.610 & 0.583 & .86 \\
\hline $\mathrm{X} 5, \mathrm{kPa} / \mathrm{L} / \mathrm{s}$ & -0.205 & -0.209 & .90 \\
\hline $\mathrm{Z} 5, \mathrm{kPa} / \mathrm{L} / \mathrm{s}$ & 0.787 & 0.777 & .64 \\
\hline Fres, $\mathrm{Hz}$ & 21.41 & 20.95 & .64 \\
\hline $\mathrm{AX}, \mathrm{kPa} / \mathrm{L}$ & 1.519 & 1.582 & .64 \\
\hline \multicolumn{4}{|l|}{ Age 7 y } \\
\hline $\mathrm{R} 5, \mathrm{kPa} / \mathrm{L} / \mathrm{s}$ & 0.717 & 0.725 & .75 \\
\hline $\mathrm{R} 20, \mathrm{kPa} / \mathrm{L} / \mathrm{s}$ & 0.513 & 0.571 & .25 \\
\hline $\mathrm{X} 5, \mathrm{kPa} / \mathrm{L} / \mathrm{s}$ & -0.244 & -0.228 & .60 \\
\hline $\mathrm{Z} 5, \mathrm{kPa} / \mathrm{L} / \mathrm{s}$ & 0.760 & 0.762 & .75 \\
\hline Fres, $\mathrm{Hz}$ & 21.44 & 19.79 & .91 \\
\hline $\mathrm{AX}, \mathrm{kPa} / \mathrm{L}$ & 1.887 & 1.598 & .91 \\
\hline \multicolumn{4}{|l|}{ Age 8 y } \\
\hline $\mathrm{R} 5, \mathrm{kPa} / \mathrm{L} / \mathrm{s}$ & 0.690 & 0.773 & .46 \\
\hline $\mathrm{R} 20, \mathrm{kPa} / \mathrm{L} / \mathrm{s}$ & 0.517 & 0.503 & $>.99$ \\
\hline $\mathrm{X} 5, \mathrm{kPa} / \mathrm{L} / \mathrm{s}$ & -0.205 & -0.238 & .25 \\
\hline $\mathrm{Z} 5, \mathrm{kPa} / \mathrm{L} / \mathrm{s}$ & 0.702 & 0.810 & .46 \\
\hline Fres, $\mathrm{Hz}$ & 20.42 & 21.65 & .60 \\
\hline $\mathrm{AX}, \mathrm{kPa} / \mathrm{L}$ & 1.497 & 2.213 & .46 \\
\hline \multicolumn{4}{|l|}{ Age $9 y$} \\
\hline $\mathrm{R} 5, \mathrm{kPa} / \mathrm{L} / \mathrm{s}$ & 0.643 & 0.661 & .60 \\
\hline $\mathrm{R} 20, \mathrm{kPa} / \mathrm{L} / \mathrm{s}$ & 0.487 & 0.487 & .89 \\
\hline $\mathrm{X} 5, \mathrm{kPa} / \mathrm{L} / \mathrm{s}$ & -0.175 & -0.203 & .51 \\
\hline $\mathrm{Z} 5, \mathrm{kPa} / \mathrm{L} / \mathrm{s}$ & 0.660 & 0.693 & .51 \\
\hline Fres, $\mathrm{Hz}$ & 18.53 & 19.89 & .15 \\
\hline $\mathrm{AX}, \mathrm{kPa} / \mathrm{L}$ & 1.108 & 1.446 & .24 \\
\hline \multicolumn{4}{|l|}{ Age $10 y$} \\
\hline $\mathrm{R} 5, \mathrm{kPa} / \mathrm{L} / \mathrm{s}$ & 0.564 & 0.458 & .08 \\
\hline $\mathrm{R} 20, \mathrm{kPa} / \mathrm{L} / \mathrm{s}$ & 0.466 & 0.384 & .07 \\
\hline $\mathrm{X} 5, \mathrm{kPa} / \mathrm{L} / \mathrm{s}$ & -0.153 & -0.133 & .22 \\
\hline $\mathrm{Z} 5, \mathrm{kPa} / \mathrm{L} / \mathrm{s}$ & 0.585 & 0.477 & .08 \\
\hline Fres, $\mathrm{Hz}$ & 15.70 & 14.18 & .29 \\
\hline $\mathrm{AX}, \mathrm{kPa} / \mathrm{L}$ & 0.717 & 0.546 & .25 \\
\hline \multicolumn{4}{|l|}{ Age $11 \mathrm{y}$} \\
\hline $\mathrm{R} 5, \mathrm{kPa} / \mathrm{L} / \mathrm{s}$ & 0.500 & 0.440 & .18 \\
\hline $\mathrm{R} 20, \mathrm{kPa} / \mathrm{L} / \mathrm{s}$ & 0.417 & 0.372 & .27 \\
\hline $\mathrm{X} 5, \mathrm{kPa} / \mathrm{L} / \mathrm{s}$ & -0.106 & -0.128 & .14 \\
\hline $\mathrm{Z} 5, \mathrm{kPa} / \mathrm{L} / \mathrm{s}$ & 0.511 & 0.459 & .20 \\
\hline Fres, $\mathrm{Hz}$ & 14.85 & 13.77 & .56 \\
\hline $\mathrm{AX}, \mathrm{kPa} / \mathrm{L}$ & 0.489 & 0.513 & .84 \\
\hline \multicolumn{4}{|l|}{ Age $12 y$} \\
\hline $\mathrm{R} 5, \mathrm{kPa} / \mathrm{L} / \mathrm{s}$ & 0.472 & 0.458 & .75 \\
\hline $\mathrm{R} 20, \mathrm{kPa} / \mathrm{L} / \mathrm{s}$ & 0.472 & 0.402 & .96 \\
\hline $\mathrm{X} 5, \mathrm{kPa} / \mathrm{L} / \mathrm{s}$ & -0.141 & -0.071 & .62 \\
\hline $\mathrm{Z} 5, \mathrm{kPa} / \mathrm{L} / \mathrm{s}$ & 0.494 & 0.479 & .75 \\
\hline Fres, $\mathrm{Hz}$ & 14.43 & 11.68 & .23 \\
\hline $\mathrm{AX}, \mathrm{kPa} / \mathrm{L}$ & 0.589 & 0.708 & .89 \\
\hline
\end{tabular}

Table 3. Continued

\begin{tabular}{|c|c|c|c|}
\hline IOS Parameters & Boy & Girl & $P$ \\
\hline \multicolumn{4}{|l|}{ Age 13 y } \\
\hline $\mathrm{R} 5, \mathrm{kPa} / \mathrm{L} / \mathrm{s}$ & 0.412 & 0.427 & .74 \\
\hline $\mathrm{R} 20, \mathrm{kPa} / \mathrm{L} / \mathrm{s}$ & 0.337 & 0.362 & .40 \\
\hline $\mathrm{X} 5, \mathrm{kPa} / \mathrm{L} / \mathrm{s}$ & -0.097 & -0.098 & $>.99$ \\
\hline $\mathrm{Z} 5, \mathrm{kPa} / \mathrm{L} / \mathrm{s}$ & 0.424 & 0.239 & .84 \\
\hline Fres, $\mathrm{Hz}$ & 12.58 & 12.94 & .94 \\
\hline $\mathrm{AX}, \mathrm{kPa} / \mathrm{L}$ & 0.404 & 0.422 & .65 \\
\hline \multicolumn{4}{|l|}{ Age 14 y } \\
\hline $\mathrm{R} 5, \mathrm{kPa} / \mathrm{L} / \mathrm{s}$ & 0.379 & 0.410 & .24 \\
\hline $\mathrm{R} 20, \mathrm{kPa} / \mathrm{L} / \mathrm{s}$ & 0.316 & 0.376 & .24 \\
\hline $\mathrm{X} 5, \mathrm{kPa} / \mathrm{L} / \mathrm{s}$ & -0.116 & -0.102 & .77 \\
\hline $\mathrm{Z} 5, \mathrm{kPa} / \mathrm{L} / \mathrm{s}$ & 0.398 & 0.422 & .24 \\
\hline Fres $\mathrm{Hz}$ & 13.05 & 10.34 & .14 \\
\hline $\mathrm{AX}, \mathrm{kPa} / \mathrm{L}$ & 0.454 & 0.277 & .77 \\
\hline $\begin{array}{l}P \text { value was from the } \\
\text { IOS }=\text { impulse oscillc } \\
\text { R5 }=\text { resistance at } 5 \\
\text { R20 }=\text { resistance at } 2 \\
\text { X5 }=\text { reactance at } 5 \\
\mathrm{Z} 5=\text { impedance at } 5 \\
\text { Fres }=\text { resonant frequ } \\
\mathrm{AX}=\text { reactance curve }\end{array}$ & ey test. & & \\
\hline
\end{tabular}

Table 4. Pearson's Correlation for Resistance at $5 \mathrm{~Hz}$, Resistance at $20 \mathrm{~Hz}$, Reactance at $5 \mathrm{~Hz}$, Impedance at $5 \mathrm{~Hz}$, Resonant Frequency, and Reactance Curve Area Below Zero for Boys and Girls

\begin{tabular}{|c|c|c|c|c|}
\hline \multirow{2}{*}{ IOS Parameters } & \multicolumn{3}{|c|}{$\mathrm{r}$} & \multirow{2}{*}{$P^{*}$} \\
\hline & Age & Height & Weight & \\
\hline \multicolumn{4}{|l|}{ Boys } & .001 \\
\hline $\mathrm{R} 5(\mathrm{kPa} / \mathrm{L} / \mathrm{s})$ & -0.72 & -0.79 & -0.73 & \\
\hline $\mathrm{R} 20(\mathrm{kPa} / \mathrm{L} / \mathrm{s})$ & -0.66 & -0.69 & -0.68 & \\
\hline $\mathrm{X} 5(\mathrm{kPa} / \mathrm{L} / \mathrm{s})$ & 0.33 & 0.39 & 0.35 & \\
\hline $\mathrm{Z} 5(\mathrm{kPa} / \mathrm{L} / \mathrm{s})$ & -0.66 & -0.71 & -0.65 & \\
\hline Fres $(\mathrm{Hz})$ & -0.69 & -0.71 & -0.63 & \\
\hline $\mathrm{AX}(\mathrm{kPa} / \mathrm{L})$ & -0.68 & -0.76 & -0.68 & \\
\hline \multicolumn{4}{|l|}{ Girls } & .00 \\
\hline $\mathrm{R} 5(\mathrm{kPa} / \mathrm{L} / \mathrm{s})$ & -0.71 & -0.75 & -0.69 & \\
\hline $\mathrm{R} 20(\mathrm{kPa} / \mathrm{L} / \mathrm{s})$ & -0.65 & -0.76 & -0.71 & \\
\hline $\mathrm{X} 5(\mathrm{kPa} / \mathrm{L} / \mathrm{s})$ & 0.69 & 0.74 & 0.62 & \\
\hline $\mathrm{Z} 5(\mathrm{kPa} / \mathrm{L} / \mathrm{s})$ & -0.72 & -0.77 & -0.70 & \\
\hline Fres $(\mathrm{Hz})$ & -0.64 & -0.61 & -0.55 & \\
\hline $\mathrm{AX}(\mathrm{kPa} / \mathrm{L})$ & -0.70 & -0.69 & -0.69 & \\
\hline \multicolumn{5}{|c|}{$\begin{array}{l}P \text { value was from the Pearson correlation test. } \\
* \text { Significance value, } P<.01 \text {. }\end{array}$} \\
\hline \multicolumn{4}{|c|}{$\begin{array}{l}\text { * Significance value, } P<.01 . \\
\text { IOS = impulse oscillometry system }\end{array}$} & \\
\hline \multicolumn{4}{|c|}{$\mathrm{R} 5=$ resistance at 5 hertz } & \\
\hline \multicolumn{4}{|c|}{$\mathrm{R} 20=$ resistance at 20 hertz } & \\
\hline \multicolumn{5}{|c|}{$\mathrm{X} 5=$ reactance at 5 hertz } \\
\hline \multicolumn{5}{|c|}{$\mathrm{Z} 5=$ impedance at 5 hertz } \\
\hline \multicolumn{5}{|c|}{ Fres $=$ resonant frequency } \\
\hline $\mathrm{AX}=$ reactance curve & w zero & & & \\
\hline
\end{tabular}



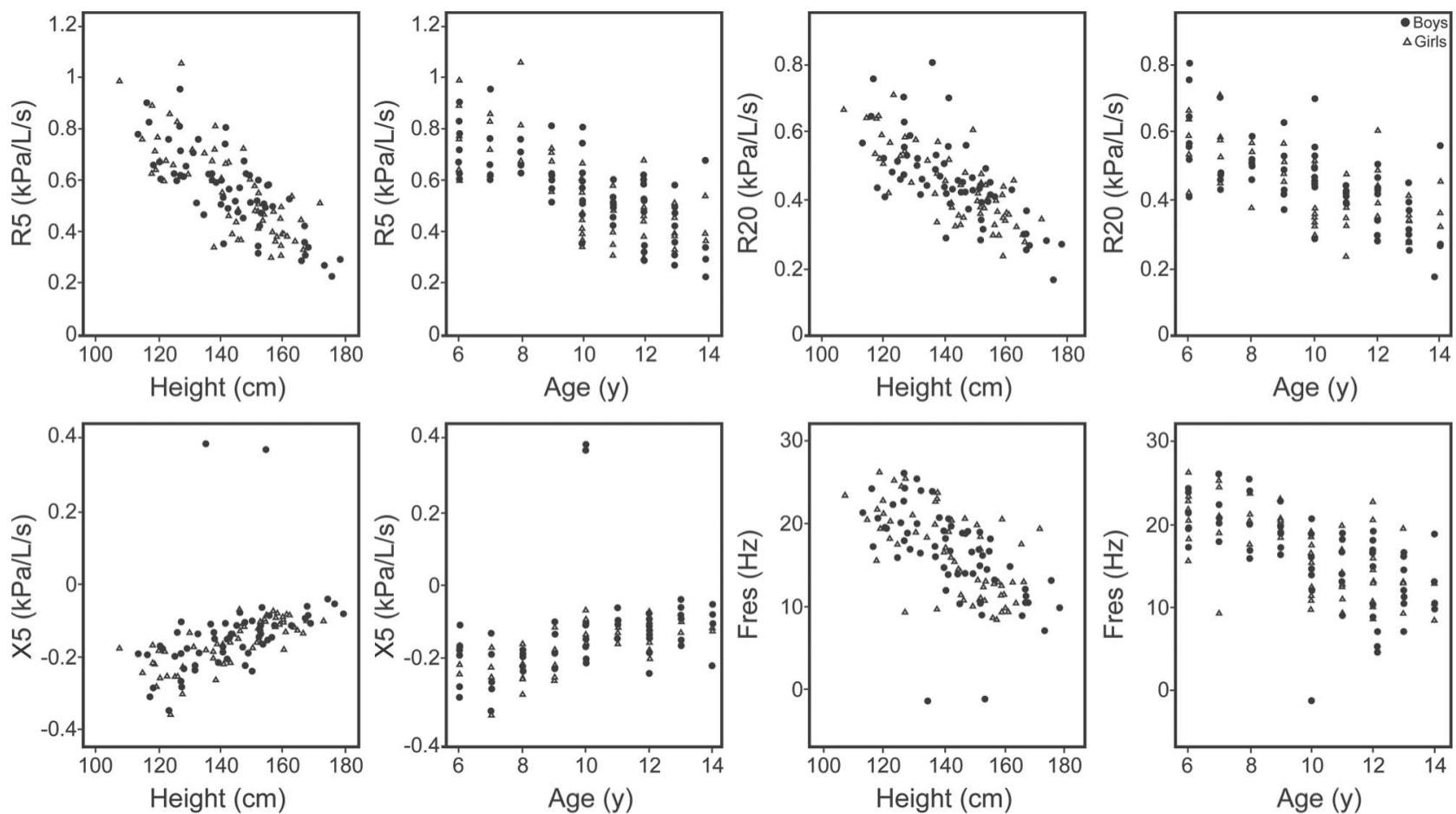

Fig. 2. Association of selected impulse oscillometry parameters with height and age. $\mathrm{R} 5=$ resistance at $5 \mathrm{~Hz}, \mathrm{R} 20=$ resistance at $20 \mathrm{~Hz}$, $\mathrm{X} 5=$ reactance at $5 \mathrm{~Hz}$, Fres $=$ resonant frequency.

Table 5. Respiratory System Reference Equations Obtained by the Impulse Oscillometry System for Boys and Girls

\begin{tabular}{|c|c|c|c|c|}
\hline IOS Parameters & Equations & $\mathrm{R}^{2}, \%$ & Adjusted $\mathrm{R}^{2}$ & SEE \\
\hline \multicolumn{5}{|l|}{ Boys } \\
\hline $\mathrm{R} 5(\mathrm{kPa} / \mathrm{L} / \mathrm{s})$ & $=1.699-(0.008 \times$ height $)$ & 62 & 61 & 0.099 \\
\hline $\mathrm{R} 20(\mathrm{kPa} / \mathrm{L} / \mathrm{s})$ & $=1.226-(0.005 \times$ height $)$ & 49 & 47 & 0.089 \\
\hline $\mathrm{X} 5(\mathrm{kPa} / \mathrm{L} / \mathrm{s})$ & $=-0.552+(0.003 \times$ height $)$ & 15.9 & 14.4 & 0.108 \\
\hline $\mathrm{Z} 5(\mathrm{kPa} / \mathrm{L} / \mathrm{s})$ & $=1.802-(0.010 \times$ height $)$ & 51 & 50 & 0.136 \\
\hline Fres $(\mathrm{Hz})$ & $=44.473-(0.195 \times$ height $)$ & 50.5 & 49.7 & 3.143 \\
\hline $\log \mathrm{AX}(\mathrm{kPa} / \mathrm{L})$ & $=0.332-(0.002 \times$ height $)$ & 58 & 57 & 0.024 \\
\hline \multicolumn{5}{|l|}{ Girls } \\
\hline $\log \mathrm{R} 5(\mathrm{kPa} / \mathrm{L} / \mathrm{s})$ & $=0.639-(0.006 \times$ height $)$ & 57.5 & 56.8 & 0.088 \\
\hline $\log \mathrm{R} 20(\mathrm{kPa} / \mathrm{L} / \mathrm{s})$ & $=0.378-(0.005 \times$ height $)$ & 59.1 & 58.4 & 0.069 \\
\hline $\mathrm{X} 5(\mathrm{kPa} / \mathrm{L} / \mathrm{s})$ & $=-0.586+(0.003 \times$ height $)$ & 55.1 & 54.1 & 0.043 \\
\hline $\log \mathrm{Z} 5(\mathrm{kPa} / \mathrm{L} / \mathrm{s})$ & $=0.679-(0.007 \times$ height $)$ & 59.9 & 59.2 & 0.086 \\
\hline Log Fres $(\mathrm{Hz})$ & $=1.555-(0.037 \times$ age $)$ & 41.1 & 40.1 & 0.109 \\
\hline $\log \mathrm{AX}(\mathrm{kPa} / \mathrm{L})$ & $=0.892-(0.106 \times$ age $)$ & 49.7 & 48.8 & 0.265 \\
\hline $\begin{array}{l}\text { IOS }=\text { impulse oscillometry s. } \\
\text { SEE }=\text { SE of the estimate } \\
\text { R5 }=\text { resistance at } 5 \mathrm{~Hz} \\
\text { R20 }=\text { resistance at } 20 \mathrm{~Hz} \\
\text { X5 }=\text { reactance at } 5 \mathrm{~Hz} \\
\mathrm{Z} 5=\text { impedance at } 5 \mathrm{~Hz} \\
\text { Fres }=\text { resonant frequency } \\
\mathrm{AX}=\text { reactance curve area be }\end{array}$ & & & & \\
\hline
\end{tabular}

Based on the highest adjusted $\mathrm{R}^{2}$ values, height and age were the variables that remained in the proposed regression models. The regression lines for boys and girls ob- tained in the present study and the other reference equations for both sexes are presented in Figure 3, considering the ideal (50th percentile). 

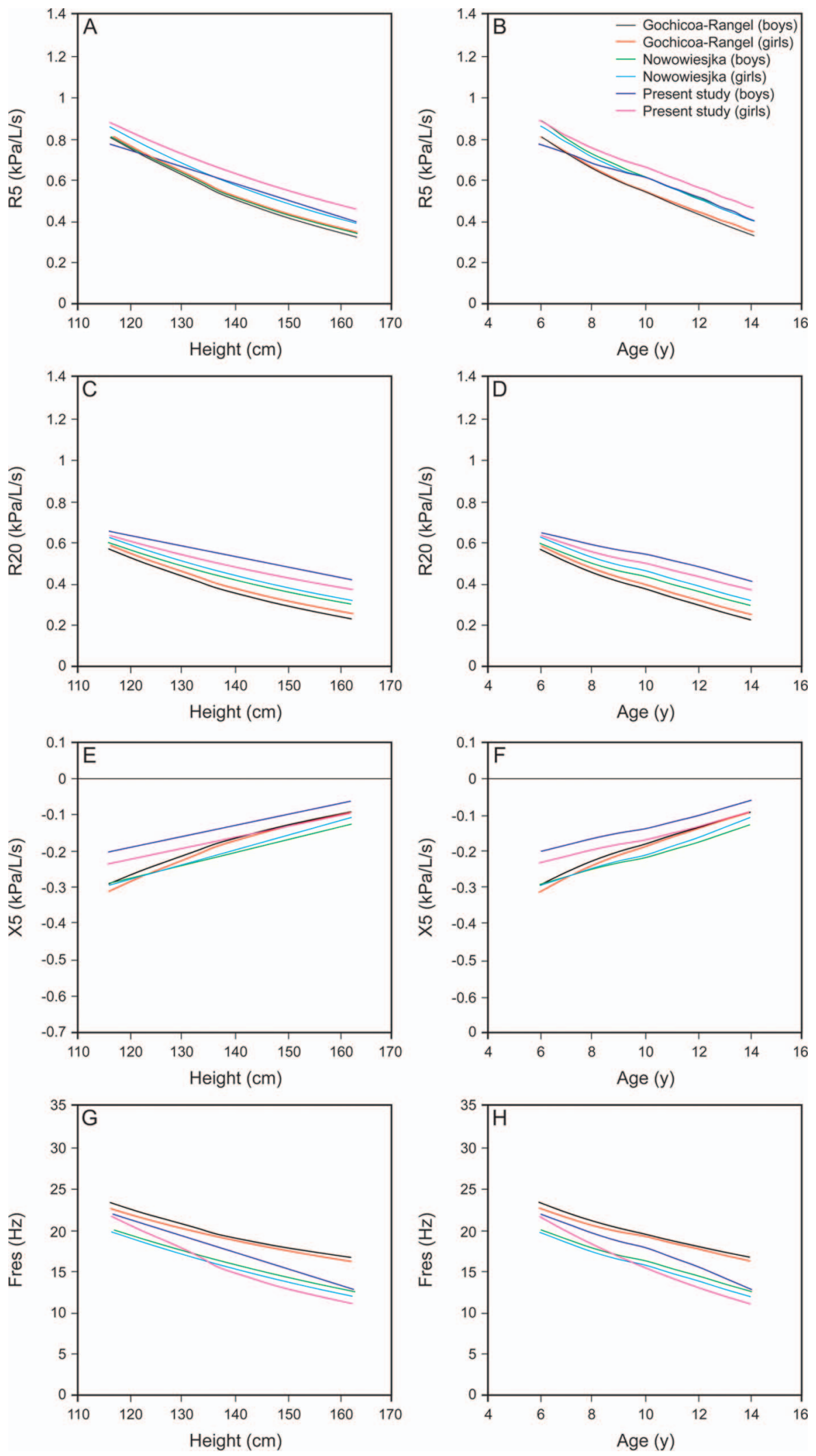

Fig. 3. Regression lines obtained in the present study and the other reference equations for boys and girls. ${ }^{6,14} \mathrm{R} 5=$ resistance at $5 \mathrm{~Hz}$, $\mathrm{R} 20=$ resistance at $20 \mathrm{~Hz}, \mathrm{X} 5=$ reactance at $5 \mathrm{~Hz}$, Fres = resonant frequency. 


\section{REFERENCE Equations For IMPUlse Oscillometry}

\section{Discussion}

This study included a sample of healthy Brazilian children and adolescents age 6-14 y, and the reference equations for the impulse oscillometry system were obtained considering the oscillometric parameters measured at 5 and $20 \mathrm{~Hz}$. To date, there are few publications on the subject in different countries. More recent work identified by a review by Assumpção et al ${ }^{31}$ include the studies by Park et al, ${ }^{4}$ Dencker et al, ${ }^{20}$ Amra et al, ${ }^{22}$ Frei et al, ${ }^{24}$ Kalhoff et al, ${ }^{32}$ and Wee et $\mathrm{al}^{33}$. In 2015, most importantly, the study by GochicoaRangel et al ${ }^{14}$ was carried out for the Latin American population.

In the development of effective equations and reference values for the different oscillometric parameters, predictor variables, such as weight, age, height, body mass index, and body surface area, have often been investigated. Among them, body surface area is the only variable that has not been shown to influence the parameters obtained by the system. ${ }^{23}$ Height has been identified as the best predictor factor, possibly because it is associated with a linear increase in lung volume. ${ }^{4,6,20,24,33,34}$ Similarly to the above mentioned studies, the reference equations obtained by the impulse oscillometry system in healthy children and adolescents in Brazil revealed that height is the variable with the greatest predictive power among the oscillometric parameters.

Height was the predominant predictor variable in impulse oscillometry system parameters in boys, which may be explained by the fact that males have larger lung volumes than females. According to the literature, males also present a disproportionate increase in chest dimensions as they grow. ${ }^{35}$ Increased respiratory muscle strength is another male characteristic that may be responsible for higher dynamic compression of the airways, evidenced by the lower means presented by boys for $\mathrm{FEV}_{1} / \mathrm{FVC} .^{36}$ These sex differences led us to develop different equations for boys and girls for each of the impulse oscillometry system parameters. The highest mean $\mathrm{FEV}_{1}$ was identified in boys $(98.60 \pm 10.17$ vs $94.63 \pm 9.55)$ when they showed greater height than girls (at ages 11-14 y). Most oscillometric parameters investigated in the present study showed agreement with the results of other publications. ${ }^{4,6,37}$ Results of the correlations between oscillometric and anthropometric variables presented the same behavior pattern, with height, age, and weight showing negative correlations for R5, R20, $\mathrm{Z} 5$, Fres, and $\mathrm{AX}$ and positive correlations with $\mathrm{X} 5$.

Also as expected, resistance (R) tends to decrease with increasing height and age. Such behavior is attributed to the growth of the child, because resistance is closely linked to distention of the pulmonary parenchyma and the phenomenon of air passage through the bronchi and bronchioles. ${ }^{38}$ Therefore, taller and older schoolchildren have increased airway dimension and, consequently, lower resistance, due to their greater thoracic size. ${ }^{6,20}$ This behavior may also be associated with Poiseuille's law. ${ }^{39}$

Another parameter that presents significant increase with height increase is the respiratory reactance $(\mathrm{X})$. The reason for this is that taller individuals have different elastic properties or heterogeneity of central airways. ${ }^{6}$ Just as all generations of the bronchial tree are formed at birth and the number of alveoli increases during growth, height increase is linked to advancing age and reflects, in part, the increase in the number of alveoli. ${ }^{40}$ Similarly to $\mathrm{R}, \mathrm{X}$ at low frequencies, such as $5 \mathrm{~Hz}$, reflects deeper lung compartments that include peripheral units of volume and length of the lung tissues, including the thorax. ${ }^{20}$

Regarding the Fres and AX parameters, the correlation between the data and age may be due to the fact that Fres determines the AX value. The AX area was proposed by Goldman as an alternative method to assess low-frequency system capacity, considering a sum of reactance values that are graphically below Fres. Such low-frequency signals reflect more clearly the peripheral airway obstruction. ${ }^{7,24}$ In older children, the value of Fres also tends to decrease because this parameter is inversely related to the size of the airway, aside from being a graphical representation that enables the identification of obstructive disorders. ${ }^{25,41}$

Considering the relationship between the oscillometric, anatomic, physiologic, and anthropometric parameters described here, there is a need for more theoretical material exploring this relationship. With regard to the anthropometric factors, the identification of height as the main predictor variable for all oscillometric parameters in males is accompanied by the presence of the Pearson correlation coefficients ( $r=0.39$ to $r=0.79$ ), introducing coefficients higher than weight and age, and of those found in other investigations. In Frei et al, ${ }^{24}$ height showed coefficients of approximately $r=0.58$ to $r=0.69$ for X5, R5, R20, Fres, and AX. Height was also identified as the predictor variable that influenced most impulse oscillometry system parameters in girls (R5, R20, X5, and Z5). ${ }^{24}$ For Fres parameters and $\mathrm{AX}$, age was the variable with the greatest correlation coefficients, corroborating the findings of Frei et al, ${ }^{24}$ in which the relationship between this variable and Fres and $\mathrm{AX}$ was greater than for weight and height.

The study that most closely matches the findings of this research is the work of Gochicoa-Rangel et al, ${ }^{14}$ which analyzed the same age group and included a healthy Latin American population. The authors found high correlation coefficients (around 80\%), and the age and height variables were inserted into equations. In this work, the oscillometric parameters $\mathrm{R}, \mathrm{X}$, and Fres were numerically higher than the values resulting from the proposed equations in the current research, which analyzed Brazilian schoolchildren. 


\section{REFERENCE EQUATIONS FOR IMPULSE OSCILlOMETRY}

Accompanying this statistical behavior of the dependent variables, the described coefficients of determination explained the model proposed here satisfactorily and with higher values than the other publications on the subject, given that the adjusted $\mathrm{R}^{2}$ remained around $46.5 \%$ in boys and $53.2 \%$ in girls. It should be noted that the findings of this research reveal a greater influence of predictive factors on the impulse oscillometry system parameters than the results of studies of other nationalities in previous years.

Amra et al ${ }^{22}$ investigated a similar age group and found that height and age were the variables best correlated to the oscillometric parameters. It is important to emphasize that the relationship between these variables and the impulse oscillometry system data for R5 and X5 presented the lowest determination coefficients (3-27\%). In other studies, the coefficients ranged from 21 to $43 \%$ and from 13 to $30 \% .^{22,24}$

The publications described above relate to schoolchildren; however, more recent studies on the determination of equations/reference values for the impulse oscillometry system have investigated even younger populations, such as preschoolers, due to the ease of application in this age group. ${ }^{4,31,34}$ However, these studies have a limitation, which is low coefficients of determination for the variables $(\sim 20 \%))^{4,42}$

Although studies on the determination of reference equations for the impulse oscillometry system in children have included different ages, frequencies, and acquisition times, most of them have found that height is an influential element in preparing the equations. A possible limitation of this study was the extensive list of criteria that children had to meet to be considered healthy, which resulted in a smaller sample. In addition, the study was conducted in few Brazilian cities, representing the behavior of that population. The reason for this is the lack of impulse oscillometry system devices available in the country and the fact that this was a pioneer study on the topic.

\section{Conclusions}

Reference equations were developed from the impulse oscillometry system parameters of children and adolescents, considering height as the most influential predictor variable in most oscillometric parameters in such a population.

\section{REFERENCES}

1. Godfrey S, Springer C, Bar-Yishay E. Evaluating the lung function of infants. Imaj 2009;11(8):492-497.

2. Eigen H, Bieler H, Grant D, Christoph K, Terrill D, Heilman DK, et al. Spirometric pulmonary function in healthy preschool children. Am J Respir Crit Care Med 2001;163(3):619-623.

3. Beydon N, Davis SD, Lombardi E, Allen JL, Arets HG, Aurora P, et al. An official American Thoracic Society/European Respiratory Society statement: pulmonary function testing in preschool children. Am J Respir Crit Care Med 2007;175(12):1304-1345.
4. Park JH, Yoon JW, Shin YH, Jee HM, Wee YS, Chang SJ, et al. Reference values for respiratory system impedance using impulse oscillometry in healthy preschool children. Korean J Pediatr 2011; 54(2):64-68.

5. Gube M, Brand P, Conventz A, Ebel J, Goeen T, Holzinger K, et al. Spirometry, impulse oscillometry and capnovolumetry in welders and healthy male subjects. Respir Med 2009;103(9):1350-1357.

6. Nowowiejska B, Tomalak W, Radliński J, Siergiejko G, Latawiec W, Kaczmarski M. Transient reference values for impulse oscillometry for children aged 3-18 years. Pediatr Pulmonol 2008;43(12): 1193-1197.

7. Meraz EG, Homer N, Ramos C, Rodriguez L, Madrigal LR, Castillo NG. Impulse oscillometric features and respiratory system models track small airway function in children. Pract Appl Biomed Eng 2013;1(1):103-140. doi: 10.5772/52579.

8. Hagiwara S, Mochizuki H, Muramatsu R, Koyama H, Yagi H, Nishida Y. Reference values for Japanese children's respiratory resistance using the LMS method. Allergol Int 2014;63(1):113-119.

9. Moreau L, Crenesse D, Berthier F, Albertini M. Relationship between impulse oscillometry and spirometric indices in cystic fibrosis children. Acta Paediatr 2009;98(6):1019-1023.

10. Komarow HD, Skinner J, Young M, Gaskins D, Nelson C, Gergen PJ, Metcalfe DD. A study of the use of impulse oscillometry in the evaluation of children with asthma: analysis of lung parameters, order effect, and utility compared with spirometry. Pediatr Pulmonol 2012;47(1):18-26.

11. El-Nemr FM, Al-Ghndour M. Study on the use of impulse oscillometry in the evaluation of children with asthma. Menoufia Med J 2013;26(2):151-158. doi: 10.4103/1110-2098.126149.

12. Lipson DA, Holsclaw D, Imbesi G, Ferrin M, Sims M, Miller S, et al. Evidence of elevated CXCR2 agonists in stable outpatients with cystic fibrosis compared with healthy controls. J Pulm Respir Med 2013;3(3):1-5. doi: 10.4172/2161-105X.1000149.

13. Stanojevic S, Wade A, Lum S, Stocks J. Reference equations for pulmonary function tests in preschool children: a review. Pediatr Pulmonol 2007;42(10):962-72.

14. Gochicoa-Rangel L, Torre-Bouscoulet L, Martínez-Briseño D, Rodríguez-Moreno L, Cantú-González G, Vargas MH. Values of impulse oscillometry in healthy Mexican children and adolescents. Respir Care 2015;60(1):119-127.

15. Hur HY, Kwak JH, Kim HY, Jung DW, Shin YH, Han MY. A comparison between impulse oscillometry system and spirometry for spirometry for detecting airway obstruction in children. Korean J Pediatr 2008;51(8):842-847. doi: 10.3345/kjp.2008.51.8.842.

16. Solé D, Vanna AT, Yamada E, Rizzo MCV, Naspitz CK. International study of asthma and allergies in childhood (ISAAC) written questionnaire: validation of the asthma component among Brazilian children. J Investig Allergol Clin Immunol 1998;8(6):376-82.

17. Vanna AT, Yamada E, Arruda LK, Naspitz CK, Solé D. International study of asthma and allergies in childhood: validation of the rhinitis symptom questionnaire and prevalence of rhinitis in schoolchildren in São Paulo, Brazil. Pediatr Allergy Immunol 2001;12(2): 95-101.

18. Behl RK, Kashyap S, Sarkar M. Prevalence of bronchial asthma in school children of 6-13 years of age in Shimla City. Indian J Chest Dis Allied Sci 2010;52(3):145-148.

19. Brazil Telehealth Program. Ministry of www.telessaudebrasil. org.br/apps/calculadoras/?page=7. Accessed May 15, 2014.

20. Dencker M, Malmberg LP, Valind S, Thorsson O, Karlsson MK, Pelkonen A, et al. Reference values for respiratory system impedance by using impulse oscillometry in children aged 2-11 years. Clin Physiol Funct Imaging 2006;26(4):247-250.

21. Du Bois D, Du Bois EF. Clinical calorimetry: tenth paper a formula to estimate the approximate surface area if height and weight be 


\section{REFERENCE EQUATIONS FOR IMPULSE OSCILLOMETRY}

known. Arch Intern Med 1916;17(6):863-871. doi: 10.1001/ archinte.1916.00080130010002.

22. Amra B, Soltaninejad F, Golshan M. Respiratory resistance by impulse oscillometry in healthy Iranian children aged 5-19 years. Iran J Allergy Asthma Immunol 2008;7(1):25-29.

23. Malmberg LP, Pelkonen A, Poussa T, Pohianpalo A, Haahtela T, Turpeinen M. Determinants of respiratory system input impedance and bronchodilator response in healthy Finnish preschool children. Clin Physiol Funct Imaging 2002;22(1):64-71.

24. Frei J, Jutla J, Kramer G, Hatzakis GE, Ducharme FM, Davis GM. Impulse oscillometry: reference values in children 100 to $150 \mathrm{~cm}$ in height and 3 to 10 years of age. Chest 2005;128(3):1266-1273.

25. Bickel S, Popler J, Lesnick B, Eid N. Impulse oscillometry interpretation and practical applications. Chest 2014;146(3):841-847.

26. Polgar G, Weng TR. The functional development of the respiratory system. Am Rev Respir Dis 1979;120(3):625-695.

27. Miller MR, Hankinson J, Brusasco V, Burgos F, Casaburi R, Coates A, et al. Standardisation of spirometry. Eur Respir J 2005;26(2):319-338.

28. Hair JF Jr, Anderson RE, Tathan RL, Black WC. Multivariate data analysis. 5th edition. Upper Saddle River, New Jersey: Prentice Hall; 1998:148-668.

29. Freund JE, Simon GA. Applied Statistics: economy, administration and accounting. 9th edition. Porto Alegre, Brazil: Bookman; 2000: 202-536.

30. Dancey C, Reidy J. Statistics without mathematics to psychology: using SPSS for Windows. 3rd edition. Porto Alegre, Brazil: Artmed; 2006:525-608.

31. Assumpção MS, Gonçalves RM, Ferreira LG, Schivinski CIS. Impulse oscillometry system in pediatrics: review. Medicina (Ribeirão Preto) 2014;47(2):131-142.

32. Kalhoff H, Breidenbach R, Smith HJ, Marek W. Impulse oscillometry in preschool children and association with body mass index. Respirology 2011;16(1):174-179.
33. Wee YS, Kim HY, Jung DW, Park HW, Shin YHS, Han MY. Reference values for respiratory impedance using impulse oscillometry in school-aged children in Korea. Korean J Pediatr 2007;50(9):862867.

34. Lai SH, Yao TC, Liao SL, Tsai MH, Hua MC, Yeh KW, Huang JL. Reference value of impulse oscillometry in Taiwanese preschool children. Pediatr Neonatol 2015;56(3):165-170.

35. Ip MS, Karlberg EM, Karlberg JP, Luk KD, Leong JC. Lung function reference values in Chinese children and adolescents in Hong Kong: I. Spirometric values and comparison with other populations. Am J Respir Crit Care Med, 2000;162(2):424-429.

36. Parazzi PLF, Barros FAA, Schivinski CIS. Interference of growth in lung function. Pediatria Moderna 2012;48(6):17-23.

37. Ai T, Luo RH, Wang CL, Yang YJ, Bao YF, Liao HL, Lu ZR. [Determination of the lung function by impulse oscillometry in 549 healthy children in Chengdu area]. Zhonghua Er Ke Za Zhi 2007; 45(10):742-745.

38. Komarow HD, Myles IA, Uzzaman A, Metcalfe D. Impulse oscillometry in the evaluation of diseases of the airways in children. Ann Allergy Asthma Immunol 2011;106(3):191-199.

39. Dufetel P, Wazni A, Gaultier C, Derossi G, Cisse F, Martineaud JP. [Growth and ventilatory function in Black children and adolescents]. Rev Mal Respir 1995;12(2):135-143.

40. Reid LM. Lung growth in health and disease. Br J Dis Chest 1984; 78(2):113-134

41. Cardoso AP, Ferreira JMR. Impulse Oscillometry. New method for evaluation of respiratory function. Rev Por Pneumol 1998;4(2):175205.

42. Lee JY, Seo JH, Kim HY, Jung YH, Kwon JW, Kim BJ, et al. Reference values of impulse oscillometry and its utility in the diagnosis of asthma in young Korean children. J Asthma 2012;49(8): 811-816. 